\title{
Reconstructing the mixed mechanisms of health: the role of bio- and socio-markers
}

\author{
Virginia Ghiara \\ University of Kent, UK \\ Federica Russo \\ University of Amsterdam, Netherlands
}

\section{Abstract}

It is widely agreed that social factors are related to health outcomes: much research served to establish correlations between classes of social factors on the one hand and classes of disease on the other hand. However, why and how social factors are an active part in the aetiology of disease development is something that is gaining attention only recently in the health sciences and in the medical humanities. In this paper, we advance the view that, just as bio-markers help trace the causal continuum from exposure to disease development at the biological level, socio-markers ought to be introduced and studied in order to trace the social continuum from exposure to disease development. We explain how socio-markers differ from social indicators and how they can be used in combination with bio-markers in order to reconstruct the mixed mechanisms of health and disease, namely mechanisms in which both biological and social factors have an active causal role.

\section{Introduction}

In the health sciences, the relationship between socio-economic factors and health has been long studied, especially by epidemiologists and sociologists. That certain diseases and health conditions are strongly associated with certain socio-economic factors is well established and documented. In particular, considerable attention has been paid to how inequalities at the socio-economic level are mirrored by inequalities at health level, and vice-versa (House, 2002; Marmot, 2005; McLean et al., 2014). In recent years, the relationship between the biological and the social dimensions of health has been addressed from novel perspectives (Vineis, Avendano-Pabon, et al., 2017). In the field of the health sciences, ground-breaking studies such as the EU-funded project Lifepath (https://www.lifepathproject.eu/) and the research conducted at the Center For Youth Wellness in San Francisco (https://centerforyouthwellness.org/) have cast a new light on the social aspects involved in 
the aetiology of disease (Bucci, Marques, Oh, \& Harris, 2016; Purewal, Marques, Koita, \& Bucci, 2016; Vineis, Illari, \& Russo, 2017; Walker \& Carrion, 2015). In addition, new discussions in the health sciences have emerged in relation to the complex processes of disease formation and prevention (Castagné et al., 2016; Davillas, Benzeval, \& Kumari, 2017; Galea, 2007; Kelly-Irving \& Delpierre, 2017; Vineis, Chatziioannou, et al., 2017). In the medical humanities, instead, novel theoretical studies have offered insights into the interaction of the social and the biological dimensions of health and its consequences for health policies (Berkman, Glass, Brissette, \& Seeman, 2000; Kelly, Kelly, \& Russo, 2014; Kelly \& Russo, 2017). We take a broad perspective on what counts as 'medical humanities', encompassing approaches coming from the social studies, history and philosophy (of science and, specifically, of medicine), and ethics (Arnott et al., 2001).

In the health sciences, the Lifepath project is particularly worthy of attention since it is the third of a series of projects that have studied the causal relationships between health and environmental exposure through biomarkers. Together with Envirogenomarkers (www.envirogenomarkers.net/) and EXPOsOMICS (https://www.exposomicsproject.eu/), Lifepath contributes to understanding exposure and disease development using innovative methodologies and concepts (Kelly-Irving \& Delpierre, 2017; Vineis, Chadeau-Hyam, et al., 2017; Vineis \& Chadeau-Hyam, 2011; Vineis \& Perera, 2007). This line of research, given its ground-breaking character, also attracted the attention both of the medical humanities (Russo and Williamson 2012; Vineis, Illari, and Russo 2017), and of philosophy of science, (Canali 2016; Illari and Russo 2016).

In particular, Lifepath brings to the fore new philosophical and methodological questions related to the biological and the social dimensions of health. In fact, current research projects using biomarkers typically try to elucidate how socio-economic factors affect disease development at the biological level. Recently, however, the literature in medical methodology - a strand of research in the medical humanities that rigorously investigates study design, research techniques, as well as foundational issues in medical research- has been pressing on other specific conceptual issues still posing a threat to the advancement of the mechanistic understanding of this phenomenon. More specifically, the details of a mixed mechanism in which both socio-economic and biological factors play a causal role needs further specification, both conceptually and methodologically. 
In this paper, we focus on one specific conceptual issue, namely how socio-economic factors can be more than just 'indicators' for broader social or health conditions. We ask the question whether, in analogy to bio-markers, which help trace the trajectory from exposure to disease at the biological level, we can identify and study socio-markers that would work in a similar way, namely helping to trace the trajectory from exposure to disease development.

The paper is organised as follows. In section 2 and 3 we introduce the study of social determinants of health and disease, paying particular attention to how social factors are measured. We claim that, over the last few years, the biological component of the mixed mechanisms of health has been explored at a fine-grained level, whereas the social component is analysed at a rather coarse-grained level through socio-economic indicators and proxies. In section 4, we introduce the idea of causation as information transmission, which constitutes a theoretical basis for using biomarkers in the study of disease mechanisms at the molecular level. Then, by analogy with biomarkers, we introduce sociomarkers as a tool to reconstruct the trajectory from exposure to disease; this, we think, explicates the causal role of social factors in disease mechanisms. Section 5 further develops on two aspects: the mixed nature of disease mechanism and the need to study these mechanisms at the population and at the individual level. These two characteristics of disease causation lend further support to the need to introduce sociomarkers, next to biomarkers. Finally, in the conclusion we claim that sociomarkers can help to address three challenges characterising the study of mixed mechanisms of disease: (i) the discrepancy between the mechanistic understanding at the biological level and at the social level; (ii) the discrepancy between the study at the individual level of the biological components of health and the study at the population level of the social components of health; (iii) the need for all these dimensions (individual/population and social/biological) for the purposes of policy interventions.

\section{Social determinants of health}

It is widely agreed that certain diseases arise from a set of risks identified at the behavioural and social level, such as people's smoking, dietary habits and social norms (Commission on the Social Determinants of Health, 2008; Davillas \& Pudney, 2017; Hernández-Quevedo, 
Masseria, Mossialos, European Commission, \& Eurostat, 2010). It is also generally accepted that the health of populations is shaped by certain 'social determinants' like social ties, poverty, and low education (Braveman \& Gottlieb, 2014; Wilkinson \& Marmot, 2003).

Despite this broad core of agreement, however, social and behavioural factors are seldom brought into the aetiological discussion of mechanisms of health and disease. This is partly because social factors are thought to provide a classification of the parts of the population more at risk. Their relevance is associated with categorization, not explanation.

Furthermore, even when it is accepted that social factors play an active role in the development of disease, how to integrate socio-behavioural and biological variables in order to develop all-encompassing models of disease causation remains largely underdeveloped. Still now, biological and social factors are considered distinct factors, whose causal role is different and whose action is far from being integrated. This assumption is evident in the linguistic use of terms such as 'proximal' and 'distal' causes, where biological factors are proximal and thus directly affecting health and disease, whereas social factors are distal, just wider determinants of health, but not proper causes (Kelly et al., 2014, p. 309; Krieger, 2008).

And yet, the need of integrated models of disease causation, where biological and social factors are considered equally relevant to the causal understanding of health has been claimed in both camps of the health sciences (Velupillai et al., 2008; Vineis \& Kriebel, 2006) and the medical humanities (Kelly et al., 2014; Russo, 2009).

This offered a raison d'être for those epidemiological studies putting at the heart of their investigations how social factors might influence health (Galea \& Link, 2013). To give an example, Castagné et al. (2016) measured inflammation through 28 markers and related them to a number of indicators for the socio-economic position of individuals. Attempts to enhance our understanding of the social component of health, however, have been often downgraded by the increasing gap between the biological and the social research advancements. Indeed, while from the biological point of view the striking technological improvements have contributed to enhancing the mechanistic understanding of disease at the biological level (Kronfeldner, 2014), at the social level the progress has not been as fast. 
This brings us to the present, when the biological component of the mechanisms of health is explored at a fine-grained level, but the social component is still analysed at a rather coarsegrained level. Indeed, social factors at the population level are often explored by using only the traditional socio-economic position indicators.

Traditionally, socio-economic status has been measured by using three social indicators: income, occupation, and educational level. Studies based on such indicators have provided useful information, and yet they suffer from substantial limitations. The main problem concerns the kind of knowledge that can be derived from this approach: the analysis of the socio-economic indicators highlights that socio-economic factors are relevant to health, however it does not establish why this is the case, namely how social factors affect health. In other words, while these measurements can show that there are robust correlations between specific socio-economic conditions and health, they are not sufficient to uncover the mechanisms through which such socio-economic conditions affect health (Muntaner, 2013).

The rationale behind this paper is that even the more accurate description of the biological aspect of disease causation is not sufficient to fully understand the phenomena of health and disease and to address questions concerning prevention and interventions. We follow existing discussions in understanding some mechanisms of health as 'mixed mechanisms', in which both biological and social factors have an active causal role (Kelly et al., 2014; Russo, 2009). We do not look at those mechanisms operating only at the biological level (such as the development of disease due to random genetic mutations) or merely at the social level (such as the way in which, through the lack of access to healthcare, socio-economic position can cause mortality). In this paper, and in analogy with biomarkers, we contribute to the characterisation of mixed mechanisms by sketching a new way to approach the social component of health through the identification of sociomarkers at the individual level. In the literature, the definition of biomarkers is not unanimous (see for instance the International Programme on Chemical Safety (2001) and the Biomarkers Definitions Working Group (2001)). The main aspect of biomarkers is, according to us, that they are measures of something: they are always used as biological clues or measures to reconstruct the continuum from exposure to disease. In parallel, we take sociomarkers to be the corresponding social measures at the individual level that allow us to understand how socio- 
economic factors 'get under the skin' and affect the biological level - we return to this point later in section 4.2. Our approach tackles a fundamental issue associated with the study of the social realm that is not yet entirely resolved: the problem of measuring the social.

\section{Measuring the social}

The study of the social dimensions of health requires the use of some measures at the social level. How to measure social phenomena, however, is a matter of debate, especially considering that not all concepts that can be measured at the social level are alike.

Some concepts refer to certain qualitative or quantitative characteristics that things have, such as the average age at marriage in a specific country or the average female pay rate. Other concepts categorize things according to several loosely interconnected features, as in the case of 'poverty' or 'socio-economic position'.

When concepts cover specific features, the selection of the methods used to generate accurate and precise measures is more straightforward and the measures are generally direct. There is no need to develop indirect measurement procedures: in order to know how many women live in a neighbourhood, for example, researchers can directly count them.

On the contrary, when a concept is multifaced or constructed in various ways, the choice of the measurement procedures is controversial and is likely to entail the sacrifice of some of its aspects. Socio-economic position, for instance, can refer both to material and social resources and assets, and to individuals' rank or status in a social hierarchy (Krieger, 2001). The measurement procedure is in general indirect and requires the selection of indicators or indices. In this case, researchers have two options: they can either develop/choose various indicators to measure all the dimensions characterizing such a concept, or they can select more precisely only some of the features constituting the concept and measure them, accepting to leave behind the other dimensions characterizing the concept. Measurements of concepts such as socio-economic position or poverty are likely to differ greatly, depending on how they are conceptualised.

Note that this distinction between simple and multifaced concepts does not correspond to the distinction between observable and unobservable things. Although certain simple concepts can be directly observed (like a person's sex), there are cases where observation 
cannot be performed with precision (like in the case of age). In the case of young individuals applying for the international protection without proper birth documentations, for instance, physicians use measurements obtained through wrist radiographs and clavicle computerized tomography to estimate age (Sauer, Nicholson, \& Neubauer, 2016).

The fact that some concepts are not easily observable has a great impact on the social sciences, as measuring them appears challenging. Researchers tackle this problem with proxies. Unlike indicators, proxies are not supposed to represent concepts. A proxy is a measure that correlates to the unobservable or unmeasurable concept under study sufficiently highly to be used as its predictor (Sapsford, 2006, p. 145).

Some examples may clarify this point: let us consider the way in which the socio-economic status is studied in epidemiological studies. In low- and middle-income countries, given the shortcomings of available measures, the presence or absence of night-time lights visible from space is increasingly used as a proxy for the concept of socio-economic position (Chen \& Nordhaus, 2011). Amongst academic research and within Government policy in England and Wales, instead, the Free School Meal Eligibility is very often used as a proxy for socioeconomic position (Hobbs, Vignoles, \& Centre for the Economics of Education (Great Britain), 2007). Recently, in addition, scholars in the UK have also proposed the use of the council tax band as an alternative proxy (Howe et al., 2012). The number attached to the presence of night-time lights in a specific zone, the Free School Meal Eligibility and the council tax band are not supposed to structurally correspond to the concept of socioeconomic position. Rather, given the absence of available measures representing it, researchers use alternative data to obtain information about that concept.

The adequacy of measures and proxies entails, hence, different questions. As for direct and indirect measures based on indicators, researchers have to establish whether the measures correctly represent the phenomena. In other words, they have to examine the adequacy of the relation between the concept and the numerical measurement. In the case of a proxy, adequacy requires the correlation between the proxy and the phenomenon to be sufficiently high. These distinctions are illustrated in Table 1. 


\begin{tabular}{lllll}
\hline Terminology of concepts & Meaning & Example & Measures & Purpose \\
\hline $\begin{array}{l}\text { Simple concepts/Social } \\
\text { Constructs }\end{array}$ & $\begin{array}{l}\text { Concepts cover } \\
\text { specific } \\
\text { features/Multifaced } \\
\text { concepts }\end{array}$ & $\begin{array}{l}\text { Number of women } \\
\text { in a } \\
\text { workplace/Poverty }\end{array}$ & $\begin{array}{l}\text { Direct } \\
\text { measures/Set of } \\
\text { indicators }\end{array}$ & Representation \\
Observable/Unobservable & $\begin{array}{l}\text { Concepts that can } \\
\text { be directly } \\
\text { observed/Concepts } \\
\text { that cannot be } \\
\text { directly observed }\end{array}$ & Sex/Age & $\begin{array}{l}\text { Direct measures } \\
\text { or set of } \\
\text { indicators/Proxies }\end{array}$ & $\begin{array}{l}\text { Predict the } \\
\text { presence of the } \\
\text { concept under } \\
\text { study }\end{array}$ \\
\hline
\end{tabular}

Table 1: This table recaps the terms used in the literature, where they apply and their purpose.

Such an approach to measuring the social has allowed for the accumulation of a large and compelling body of evidence demonstrating that socio-economic factors are correlated to a wide range of health outcomes. The result of a meta-analysis conducted by Galea and colleagues (2011), for instance, showed that the number of U.S. deaths in 2000 correlated to socio-economic factors such as low education, racial segregation, and low social support, and that such a correlation was comparable to the number of death correlated to cerebrovascular disease and lung cancer. Although this growing weight of evidence reflects strong associations between socio-economic factors and health, the study of socioeconomic indicators and proxies does not help to uncover the causal mechanisms behind them. It follows that socio-economic factors are rarely brought into the aetiological discussion of mechanisms of health.

At the biological level, instead, much attention has been paid to eliciting the mechanisms behind correlations at the individual level, trying to understand them at an increasing level of detail. Researchers pinned down the biological and chemical basis of disease causation until the molecular level, and in these studies biomarkers play an essential role. Some interesting findings have been established in recent years through the study of biomarkers, yet without a mechanistic understanding of the social component of health, social epidemiology runs the risk of veering towards biologic reductionism (Muntaner, 2013). 


\section{Markers}

\subsection{Markers and information transmission}

In general, causal claims in social epidemiology involve statements about risks: scientists want to know whether certain socio-economic conditions are good predictors of disease. As considered above, however, researchers do not only aim at claiming that socio-economic factors are correlated to health, they want to establish (i) that such factors are causally linked to health outcomes and (ii) how they can cause health outcomes. While the recognition that there is a causal link can be obtained through different methods, typically, the 'how' question is answered by considering the causal process linking the putative causal factor to the effect at the biological level.

In similar cases, the continuum is explored by collecting information through markers. In the context of the biomedical sciences, they are most often referred to as biomarkers. Such markers are clues, signals to detect in order to understand how factors at different molecular levels can be causally linked to different stages of disease development. It is important to note that markers are not just 'objects out there'. Sometimes, they are described in terms of 'events' in the continuum from the cause to the effect (Schulte \& Perera, 1993), other times they are considered 'possible intersecting signals' representing parts of an active process (Chadeau-Hyam et al., 2011). The key feature of markers is that they are always marks of specific links, events, or processes. It is impossible to establish what objects count as markers in the abstract: markers are not defined in terms of intrinsic properties or correlations, but in terms of the function they have within particular biological processes under investigation. It follows that researchers have to decide whether something is a marker or not by considering the concrete research situations in which markers are studied, rather than on a priori set of identification criteria.

While the idea of a marker - and especially a biomarker - is most common in contemporary biomedical research, it is worth noting that it had already been introduced in the philosophy of causality since the 1980 s. Causal processes can be characterized in several different ways. One of the prominent interpretations is Wesley Salmon's 'mark transmission theory' (Dowe, 1992; Salmon, 1984). According to this account, what makes causal processes different from non-causal or pseudo-processes is the fact that causal processes are the ones transmitting 
marks. For instance, a moving car can be considered a causal process because, if we mark or dent it, such a mark will be carried along with the car, and it will remain detectable at later stages. On the contrary, if we try to mark the car's shadow (for instance interrupting the shadow by shining a light), that kind of mark will not travel with the car at later stages, therefore the mark will not be transmitted. Unlike the car's example, however, there are many situations in which a process cannot be marked: this is the reason why Salmon formulated his proposal in counterfactual terms: a process is causal if and only if, if it were marked, the mark would be transmitted to later stages of the same process.

Salmon's approach is intuitively strong but limited: it is specifically tailored to physics, and considers marks as physics quantities such as energy and momentum. Consequently, it cannot be directly used as a conceptual tool to understand causal links that cannot be spelled out with the language and tools of physics. This limitation has long been recognized in the health sciences, where researchers explicitly recognize the importance of studying the reality at different levels (such as the biological and the social levels), for instance using a longitudinal approach, where marks are observed rather than introduced (Vineis, 1997).

However, an adjustment can be made to tackle this problem: a marker might be something that is already transmitted by a causal process, rather than a 'physics' mark that should be introduced. This would appear in line with what is done in epidemiological studies (such as in the aforementioned Lifepath, EXPOsOMICS, or Envirogenomarkers), where researchers follow a longitudinal thread of marks (observing, for instance, that aflatoxin causes the formation of adducts, that in turn induce p53 mutations that are the same found in liver cancer). In the philosophy of causation and philosophy of medicine literature, it has been recently suggested that the link shown by markers is better conceptualized using the notion of “information transmission” (Illari \& Russo, 2016; Vineis, Illari, et al., 2017).

Thus, markers can be conceptualized as signals of transmission of information. It is important to note that the notion of information transmission is located at the level of epistemology, not of ontology: information transmission deals with the way in which scientists reconstruct the continuum between factors at different levels (Vineis, Illari, \& Russo, 2017). This proposal is not necessarily in contrast with the prominent mechanistic thinking now widespread in philosophy of causality and in the sciences: when looking for markers, indeed, researchers might be guided by mechanistic knowledge. Illari and Russo 
(2016) described mechanisms as information channels where information flows and markers (i.e. the intersecting signals) can be found.

The generality of the information-transmission idea, and of how markers help (re)trace the link from putative causes to putative effects, allows for the development of a general epistemological framework for thinking about causality across different levels (individual, population) and across different types of factors (biological, social). For this reason, understanding causality as information transmission enables us to provide a mechanistic explanation of disease that includes both biological and social factors.

It remains to explain, however, how, more specifically, markers can help detect links at the biological level, and even at the social level. This is what the following two sections aim to do. At the biological level, the development of new technologies, as well as the availability of large datasets, led to an increasing use of biological markers. In parallel, we argue it is high time to approach social measures not as 'indicators' and 'proxies', but as sociomarkers.

\subsection{Biomarkers}

The striking technological advancements of the last few decades have contributed to enhancing the mechanistic understanding of disease from a biological point of view. The main protagonists of this advancement are the so-called '-omics' technologies and their products, known by the name of biological markers, or biomarkers. One of the major contributions of '-omics' technologies is through the identification of relevant markers at the molecular level. Traditionally, epidemiology established correlations between macrovariables: those associated with the environment and those describing diseases. Biomarkers, instead, allow researchers to go much smaller: both the macro-level factors themselves, such as air pollution, and their effects can now be measured at the molecular level.

Biomarkers do not necessarily correspond to specific molecular entities. Often, they are constructed by cross-checking large datasets and using background information about the processes that biomarkers are supposed to signal. They are not studied per se, or to identify those entities causing health effects, but to understand the trajectory of disease development. They can be conceptualized as the 'missing links' between factors at the 
population (macro) level (such as external or environmental exposure) and factors at the individual (micro) level (such as DNA alterations leading to disease). By helping to detect some key stages linking such factors, biomarkers suggest the existence of a continuum between them.

Biomarkers can mark salient points in the continuum from exposure to disease in several ways, consequently they can stand in different relationships with each other, the effect and the cause. Diagrams consisting in variables linked by arrows or lines, also known by the name of Directed Acyclic Graphs (DAGs), can be used to illustrate relationships that are relatively complicated and larger causal system based on inter-relationships relevant to health (Joffe, Gambhir, Chadeau-Hyam, \& Vineis, 2012). In Figure 1, we illustrate most possible linkages between the biomarker and the phenomenon under study through the use of DAGs, where arrows represent causal relationships and lines simple correlations.

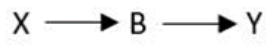

1(a)

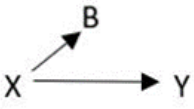

1(b)

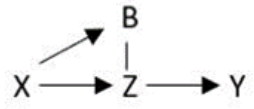

1(c)

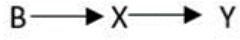

1(d)

Figure 1: (a) represents the situation in which the biomarkers $B$ is part of the causal process linking the cause $X$ and the effect $Y$. In (b), the biomarker $B$ and the effect $Y$ are correlated because both are caused by $X . \operatorname{In}(c)$, the biomarker $B$ is correlated to the event $Z$, that is part of the process but cannot be measured. In (d), B is just correlated and is not causally linked to the effect $Y$. B may be, for instance, a background condition.

Biomarkers are conceived and used to mark processes that happen at the biological level, reconstructing the link between exposure and disease. They shed light on several aspects of disease mechanisms at the molecular level, and contribute to early diagnosis and better prediction. However, disciplines such as social epidemiology or medical sociology have long established and pleaded for the important role of social factors for health (Braveman \& Gottlieb, 2014). What remains to understand, is whether there is any way to shed light on the social mechanisms of health at the individual level. This is what we explore next.

\subsection{Sociomarkers}


Rather than being considered competing approaches, the individual and population level can be reconciled through the study of sociomarkers: it is the analysis of sociomarkers at the individual level that can enhance our understanding of the social components of health at the population level.

Traditionally, social epidemiology establishes correlations between health outcomes and population-level factors such as poverty and socio-economic position. On the contrary, sociomarkers can be used to trace social processes at the individual level, helping to find the missing link between social factors at the population level and health factors at the individual level.

It is important to note that the shift from the notion of social indicators to the concept of sociomarkers does not necessarily require the collection of new measures. Available measures, already used as proxies and indicators, might count as markers. It is not the particular nature of measures, but how they are used that determines whether something is a sociomarker. The aim, when using sociomarkers, is to pick up signals to reconstruct the continuum from social factors to disease, in analogy with how biomarkers help pick up signals from exposure to disease at the biological level. In practice, this might require using socio-economic variables measured at the individual level, rather than aggregate level. This does not exclude the use of aggregate variables or of multi-level methods that use both population- and individual-level variables. In some cases, for instance, it could be possible to use population-level sociomarkers that describe the role of structural causes (this would go into the same direction as Galea and Link's, according to which epidemiology is 'all social' (Galea \& Link, 2013, p. 844)). It does suggest, however, how else individual-level variables that measure socio-demo-economic characteristics can be used. Individual-level variables, furthermore, might measure both individual behaviours (such as social interaction) and individual-level effects that depend on structural causes active at the population or macro level (such as violent behaviours caused by some structural features of society).

When measures are used as sociomarkers, they are not associated with specific concepts, and are not supposed to have a structural correspondence with them, like indicators. Sociomarkers are always located in relation to the causal continuum. As biomarkers, they might be part of the continuum, or might be simply correlated to the continuum, as shown in Figure $1(b)$ and (c). When sociomarkers exhibit just a difference-making relationship with 
the continuum, however, they do not act just as proxies. In causal reasoning proxies are used to establish that something (notably, what they stand for) makes a difference (for instance, using the council tax band as a proxy of poverty, we can claim that poverty exerts an effect on health), while sociomarkers help to find out how something causes the effect (for instance, individual values of council tax bands should be linked to specific individual biological outputs). The 'how', again in analogy with biomarkers, is provided by the process linking socio-economic characteristics and health outcomes, and sociomarkers help intercept this process at relevant places, just as biomarkers do. In this way sociomarkers do more than just identifying social risks - they indicate where to intervene in the whole process.

Recently, scholars have begun to use the label 'markers' for socio-economic measures in a rudimentary way. For instance, measures such as birthweight, household income, education, maternal smoking during pregnancy, mother's body mass index and mother's age at birth, known to affect later health, were used to explore how the link between socioeconomic position and health is mediated by certain social pathways (Barboza Solís et al., 2016; Davillas et al., 2017). It is exactly when such measures are used, we claim, that a precise conceptualization of sociomarkers is needed.

Let us consider an example: it is known that a low socio-economic status in early life is correlated with poor health and increased mortality in adulthood (Anda et al., 2006). At the social level, a general hypothesis is that socio-economic factors in childhood get under the skin (at the molecular level) through severe chronic stressors to which children are exposed (Miller, Chen, \& Parker, 2011).

To detect signals of this process at the social level, some researchers have used the so-called Adverse Childhood Experiences (ACEs). This expression describes certain stressful or traumatic events that can be experienced by children whilst growing up. The original ACEs study was conducted by the Centers for Disease Control and Prevention (CDC) in collaboration with the American integrated managed care consortium Kaiser Permanente (Felitti et al., 1998). In this study, researchers developed a list of 10 indicators of child adverse experiences: emotional abuse, physical abuse, emotional neglect, physical neglect, contact sexual abuse, mother treated violently, alchoolic or drug users among the household members, not being raised by both biological parents, household member who 
was imprisoned, household member who was chronically depressed, suicidal, mentally ill or in a psychiatric hospital.

The original ACEs, as well as the new ACEs added in the literature such as separation from family and serious childhood illness (Hughes et al., 2017), have all the characteristics required to be used as sociomarkers. First, it is possible to use ACEs information as intersecting signals of a continuum. ACEs can indeed be employed to identify some key stages connecting social factors at the population level, such as the socio-economic position, and biological factors at the individual level (biomarkers). In such a way, ACEs are not just indicators, but sociomarkers that can help to establish how socio-economic determinants exert an impact on health through biological mechanisms. This is possible for two reasons. One the one hand, at the population level, ACEs appear to be more prevalent among children in low-income families: in the U.S., for instance, data from the National Survey of Children's Health (NSCH) showed that children in low- and middle-income families are more than twice as likely to experience two or more ACEs (Halfon, Larson, Son, Lu, \& Bethell, 2017). This prevalence, hence, supports the idea that socio-economic conditions during childhood could affect health through these adverse experiences. On the other hand, several studies have provided evidence of the associations between ACEs and allostatic load biomarkers, inflammatory biomarkers and cancer biomarkers (Barboza Solís et al., 2015; Kelly-Irving et al., 2013; Tietjen, Khubchandani, Herial, \& Shah, 2012). Such correlations support the idea that ACEs could be the link connecting 'the social' and 'the biological', as described by Danese and McEwen (2012):

"adverse childhood experiences are likely to signal high levels of environmental threat and to trigger adaptive responses in the nervous, endocrine, and immune systems in children" (Danese \& McEwen, 2012, p. 31)

Second, in contrast to traditional socio-economic indicators, ACEs help to investigate the processes from social events to biological changes at the individual level. Like biomarkers, ACEs are identified by examining real individuals: ACEs can be experienced only by real, individual children. This is particularly relevant if we consider that for each individual adverse childhood experiences can vary in duration, timing, and type, and that individual characteristics can lead to very different responses to very similar adversities. In this and similar cases, hence, sociomarkers would help us to capture some aspects of the 
subjective/reflexive/perceived dimension of the social: the assumption justifying the use of ACEs would be that events such as the incarceration of a parent can be associated with an individual, perceived dimension, that can in turn influence the individual's health.

\section{Types of markers and levels of causation}

In section 4.2 we suggested that, next to biomarkers, we need to study sociomarkers too. Bio- and socio-markers help with identifying/detecting the process from exposure (biological and social) to health outcomes at two levels: (i) the biological and social level, and (ii) the individual and population level. Concerning the first, this is how we can shed light on the mixed mechanisms of health and disease - we address this further in section 5.1. Concerning the second, earlier in section 3 we noticed that measurements of the social still pertain to the population level. However, with the advent of molecular approaches, even epidemiology could reach the individual level. How do these two level relate to each other? We address this further in section 5.2.

\subsection{Bio-social mechanisms of disease}

Our proposal is that socio- and bio-markers should be used in combination. In such a way it would be possible to finally uncover the mixed, bio-social mechanisms whereby 'the social' can influence 'the biological', returning to a more holistic understanding of the aetiology of disease (Kelly et al., 2014; Russo, 2009).

In the current situation, the biological component of the mechanisms of disease is explored at a fine-grained level (explanations of disease at the molecular level are paradigmatic in this respect), while the social component is analysed at a rather coarse-grained level, or at the level of population using mainly aggregate variables. As a consequence, the mechanisms of disease appear to be spelled out only in biological terms, while the study of the social components allows researchers only to establish that there are robust correlations between specific social factors and some biological outcomes. In line with recent arguments of Kelly et al. (2014), and Kelly and Russo (2017) we aim to put the social in the mechanisms explaining disease.

The combination of biomarkers and sociomarkers, we argue, would finally help researchers uncover the mixed mechanisms through which 'the social' affects health. To begin with, 
sociomarkers would fill the gap between biological fine-grained variables, and social coarsegrained variables, enabling researchers to study both the biological and the social components at a fine-grained level. Together, biomarkers and sociomarkers would enable researchers to sample the different levels of reality at different time points, and the passages from specific sociomarkers to certain biomarkers would provide some compelling linkages between these different domains. Given that the continuum traced through these markers could involve both social and biological components, it follows that such markers would allow for the identification of the key stages of a mixed mechanism of disease, from the social components triggering the mechanism, to certain key events involving social or biological components, to the biological factors leading to the health outcome.

Sociomarkers would hence help us recognize, despite their 'distance from the skin', the epistemic importance of 'the social' for the mixed mechanism of disease; and sociomarkers and biomarkers together would lead us to reconceptualize, from a mechanistic point of view, causality across different types of factors (social and biological). This means that, while bio- and sociomarkers are ontologically distinct, a priori they have equal importance in the explanation and reconstruction of exposure and disease development.

\subsection{Population and individual causation}

With biomarkers, we study processes that happen at the individual level, and molecular epidemiology aims to provide a 'population-level' study of them. On the one hand, biomarkers are searched for and validated using individual-level data - biosamples pertain to individuals; on the other hand, the fact that we can dispose of very large bio-banks or cohorts make it possible to make an epidemiological study out of it. So, if we run a study at the individual level - and even at a finer grained level (i.e. the molecular level), the ultimate goal is to say something at the population level. This, in the early days of molecular epidemiology created a tension in the field. As Schulte put it, it might sound an oxymoron to do epidemiology with molecular data (Schulte, 1993, p. 10). We agree with Schulte that this is not the case, but we are forced to re-think the relation between population- and individual-level causal knowledge, and specifically the role of biomarkers in the production of generic knowledge of health and disease. Likewise, a large part of social science interested in health has worked at the population level, while our proposal to use 
sociomarkers shifts the level of analysis to the individual level. How do we reconcile these different levels of analysis?

To begin with, it is worth clarifying the terminology used. We endorse the categorisation provided by Illari and Russo (2014), and summarised in the table below, taken from their work (2014, p. 43).

\begin{tabular}{|c|c|c|c|c|}
\hline & Meaning & Example & Used by / context & Applies to \\
\hline Population / Individual & $\begin{array}{l}\text { Refers to a causal claim either at the } \\
\text { population level or at the individual level }\end{array}$ & $\begin{array}{l}\text { Smoking causes cancer / Bob's } \\
\text { smoking caused his cancer }\end{array}$ & $\begin{array}{l}\text { Philosophical literature, probabilistic } \\
\text { causality }\end{array}$ & Causal claim \\
\hline Type / Token & $\begin{array}{l}\text { Refers to causal relations between } \\
\text { characteristics or between instantiated } \\
\text { events }\end{array}$ & $\begin{array}{l}\text { Smoking causes cancer / Bob's } \\
\text { smoking caused his cancer }\end{array}$ & $\begin{array}{l}\text { Philosophical literature, probabilistic } \\
\text { causality }\end{array}$ & Causal claim \\
\hline Generic / Single-case & $\begin{array}{l}\text { Refers to a causal relation that can be } \\
\text { instantiated more than once or to an } \\
\text { instantiated relation / Single-case causal } \\
\text { relations can also be at the population } \\
\text { level }\end{array}$ & $\begin{array}{l}\text { Smoking causes cancer / Bob's } \\
\text { smoking caused his cancer / The } \\
\text { financial crisis in } 2008 \text { caused } \\
\text { recession in Europe }\end{array}$ & $\begin{array}{l}\text { Link philosophical and scientific } \\
\text { literature }\end{array}$ & Causal claim \\
\hline Aggregate / Individual & $\begin{array}{l}\text { Summary of characteristics of individuals } \\
\text { composing a group / Measure of } \\
\text { individual characteristics, taking values of } \\
\text { each of the lower units in the sample }\end{array}$ & $\begin{array}{l}\text { Mean income of state residents / } \\
\text { Income of each individual in the } \\
\text { sample }\end{array}$ & Scientific literature & Variable \\
\hline
\end{tabular}

Table 2: This table, taken from Illari and Russo (2014, p. 43), recaps the terms used in the literature, where they apply and their purpose

Biomarkers are identified using bio-samples of real, particular patients, but their study in large data sets allows scientists to make inferences about the population, rather than single individuals. The change of the scale of measurement, the size of the sample, and the way in which inferences from -omics analyses are made allows us to make something of unprecedented novelty: to study individual-level processes in order to understand population-level phenomena.

In analogy to the study of biomarkers, we want to suggest studying sociomarkers. These sociomarkers will differ substantially from the widely used social indicators in that they allow us to trace social process at the individual level. Again, in analogy with biomarkers, we can then develop an 'epidemiology' of sociomarkers, and thus gain understanding of the social mechanisms that operate at the individual level, but within a group or population. By construction, the sought sociomarkers will be those likely to interact with the bio-chemical disease mechanisms. With sociomarkers we will be able to trace the onset and development of disease in its biological and social aspects. The equal epistemic relevance of bio- and socio- markers will help us to reconstruct the mixed mechanism of health, while taking their ontological differences into account. Knowledge of the development of sociomarkers, 
furthermore, can be used for early diagnosis and screening at the individual level, just as it happens for biomarkers.

The introduction of sociomarkers also contributes to avoiding the risk of reductionism in at least two senses. Firstly, health and disease are not reduced to the biological realm; instead, social factors are active causes in disease aetiology. Secondly, while the study of sociomarkers at the individual level help eliciting the mixed mechanisms, health and disease are not confined to the individual level; instead, individual-level sociomarkers help understand how population-level social risks act.

\section{Conclusion}

The interaction between social and biological components of health has been at the heart of several studies in the last few decades. This paper identifies the following challenges, that we try to constructively address. First, the increased understanding of the biological factors involved in the mixed mechanisms of diseases formation has not been accompanied by an appropriate comprehension of the active role played by social factors. It follows that our mechanistic understanding is in general obtained only at the biological level, while the social level is still studied through correlations between population-level variables.

Second, concerning the biological components, our mechanistic understanding can be achieved at the individual level through the use of biomarkers. In contrast, social components are still studied at the population level.

Third, all these dimensions (individual/population and social/biological) are relevant for the purposes of policy interventions. Let us consider obesity: in order to develop an intervention, researchers ought to take into account population- and individual-level social factors, such as food availability, the presence or absence of walkable neighbourhoods, and the individual lifestyle and ideal concerning body size. Similarly, they might consider macroand micro- biological factors, such as the presence of environmental factors and molecular mechanisms underlying obesity.

This paper contributes to addressing these challenges by proposing to use social measures as sociomarkers. To describe what sociomarkers are, a parallel with biomarkers has been proposed. Both social and biological markers can be understood as 'intersecting signals' and 
can be related in different ways to the causal process under study. Furthermore, in both cases markers would help to trace causal processes at the individual level.

The combination of social and biological markers, in conclusion, would reveal how social factors can "get under the skin", and would help researchers to move from causal claims at the population level to causal claims at the individual level.

\section{References}

Anda, R. F., Felitti, V. J., Bremner, J. D., Walker, J. D., Whitfield, C., Perry, B. D., ... Giles, W. H. (2006). The enduring effects of abuse and related adverse experiences in childhood: A convergence of evidence from neurobiology and epidemiology. European Archives of Psychiatry and Clinical Neuroscience, 256(3), 174-186. https://doi.org/10.1007/s00406-005-0624-4

Arnott, R., Bolton, G., Evans, M., Finlay, I., Macnaughton, J., Meakin, R., \& Reid, W. (2001). Proposal for an academic Association for Medical Humanities. Medical Humanities, 27(2), 104. https://doi.org/10.1136/mh.27.2.104

Barboza Solís, C., Fantin, R., Castagné, R., Lang, T., Delpierre, C., \& Kelly-Irving, M. (2016). Mediating pathways between parental socio-economic position and allostatic load in mid-life: Findings from the 1958 British birth cohort. Social Science \& Medicine, 165, 19-27. https://doi.org/10.1016/i.socscimed.2016.07.031

Barboza Solís, C., Kelly-Irving, M., Fantin, R., Darnaudéry, M., Torrisani, J., Lang, T., \& Delpierre, C. (2015). Adverse childhood experiences and physiological wear-and-tear in midlife: Findings from the 1958 British birth cohort. Proceedings of the National Academy of Sciences, 112(7), E738-E746. https://doi.org/10.1073/pnas.1417325112

Berkman, L. F., Glass, T., Brissette, I., \& Seeman, T. E. (2000). From social integration to health: Durkheim in the new millennium. Social Science \& Medicine, 51(6), 843-857. https://doi.org/10.1016/S0277-9536(00)00065-4

Biomarkers Definitions Working Group.. (2001). Biomarkers and surrogate endpoints: Preferred definitions and conceptual framework. Clinical Pharmacology \& Therapeutics, 69(3), 89-95. https://doi.org/10.1067/mcp.2001.113989 
Braveman, P., \& Gottlieb, L. (2014). The Social Determinants of Health: It's Time to Consider the Causes of the Causes. Public Health Reports, 129(1_suppl2), 19-31. https://doi.org/10.1177/00333549141291S206

Bucci, M., Marques, S. S., Oh, D., \& Harris, N. B. (2016). Toxic Stress in Children and Adolescents. Advances in Pediatrics, 63(1), 403-428. https://doi.org/10.1016/i.yapd.2016.04.002

Canali, S. (2016). Big Data, epistemology and causality: Knowledge in and knowledge out in EXPOsOMICS. Big Data \& Society, 3(2), 205395171666953.

\section{https://doi.org/10.1177/2053951716669530}

Castagné, R., Delpierre, C., Kelly-Irving, M., Campanella, G., Guida, F., Krogh, V., ... ChadeauHyam, M. (2016). A life course approach to explore the biological embedding of socioeconomic position and social mobility through circulating inflammatory markers. Scientific Reports, 6(1). https://doi.org/10.1038/srep25170

Chadeau-Hyam, M., Athersuch, T. J., Keun, H. C., De lorio, M., Ebbels, T. M. D., Jenab, M., ... Vineis, P. (2011). Meeting-in-the-middle using metabolic profiling - a strategy for the identification of intermediate biomarkers in cohort studies. Biomarkers, 16(1), 8388. https://doi.org/10.3109/1354750X.2010.533285

Chen, X., \& Nordhaus, W. D. (2011). Using luminosity data as a proxy for economic statistics. Proceedings of the National Academy of Sciences, 108(21), 8589-8594. https://doi.org/10.1073/pnas.1017031108

Commission on the Social Determinants of Health. (2008). Closing the Gap in a Generation: Health Equity Through Action on the Social Determinants of Health. Geneva: WHO.

Danese, A., \& McEwen, B. S. (2012). Adverse childhood experiences, allostasis, allostatic load, and age-related disease. Physiology \& Behavior, 106(1), 29-39. https://doi.org/10.1016/i.physbeh.2011.08.019

Davillas, A., Benzeval, M., \& Kumari, M. (2017). Socio-economic inequalities in C-reactive protein and fibrinogen across the adult age span: Findings from Understanding Society. Scientific Reports, 7(1). https://doi.org/10.1038/s41598-017-02888-6

Davillas, A., \& Pudney, S. (2017). Concordance of health states in couples: Analysis of selfreported, nurse administered and blood-based biomarker data in the UK Understanding Society panel. Journal of Health Economics, 56, 87-102. https://doi.org/10.1016/j.jhealeco.2017.09.010 
Dowe, P. (1992). Wesley Salmon's Process Theory of Causality and the Conserved Quantity Theory. Philosophy of Science, 59(2), 195-216. https://doi.org/10.1086/289662 Felitti, V. J., Anda, R. F., Nordenberg, D., Williamson, D. F., Spitz, A. M., Edwards, V., ... Marks, J. S. (1998). Relationship of Childhood Abuse and Household Dysfunction to Many of the Leading Causes of Death in Adults. American Journal of Preventive Medicine, 14(4), 245-258. https://doi.org/10.1016/S0749-3797(98)00017-8

Galea, S. (Ed.). (2007). Macrosocial determinants of population health. New York, NY: Springer.

Galea, S., \& Link, B. G. (2013). Six Paths for the Future of Social Epidemiology. American Journal of Epidemiology, 178(6), 843-849. https://doi.org/10.1093/aje/kwt148

Galea, S., Tracy, M., Hoggatt, K. J., DiMaggio, C., \& Karpati, A. (2011). Estimated Deaths Attributable to Social Factors in the United States. American Journal of Public Health, 101(8), 1456-1465. https://doi.org/10.2105/AJPH.2010.300086

Halfon, N., Larson, K., Son, J., Lu, M., \& Bethell, C. (2017). Income Inequality and the Differential Effect of Adverse Childhood Experiences in US Children. Academic Pediatrics, 17(7), S70-S78. https://doi.org/10.1016/i.acap.2016.11.007

Hernández-Quevedo, C., Masseria, C., Mossialos, E., European Commission, \& Eurostat. (2010). Analysing the socioeconomic determinants of health in Europe: new evidence from EU-SILC. Luxembourg: Publications Office. Retrieved from http://dx.publications.europa.eu/10.2785/5478

Hobbs, G., Vignoles, A., \& Centre for the Economics of Education (Great Britain). (2007). Is free school meal status a valid proxy for socio-economic status (in schools research)? London: Centre for the Economics of Education. Retrieved from http://cee.Ise.ac.uk/cee\%20dps/ceedp84.pdf

House, J. S. (2002). Understanding Social Factors and Inequalities in Health: 20th Century Progress and 21st Century Prospects. Journal of Health and Social Behavior, 43(2), 125. https://doi.org/10.2307/3090192

Howe, L. D., Galobardes, B., Matijasevich, A., Gordon, D., Johnston, D., Onwujekwe, O., ... Hargreaves, J. R. (2012). Measuring socio-economic position for epidemiological studies in low- and middle-income countries: a methods of measurement in epidemiology paper. International Journal of Epidemiology, 41(3), 871-886. https://doi.org/10.1093/ije/dys037 
Hughes, K., Bellis, M. A., Hardcastle, K. A., Sethi, D., Butchart, A., Mikton, C., ... Dunne, M. P. (2017). The effect of multiple adverse childhood experiences on health: a systematic review and meta-analysis. The Lancet Public Health, 2(8), e356-e366. https://doi.org/10.1016/S2468-2667(17)30118-4

Illari, P., \& Russo, F. (2014). Causality: philosophical theory meets scientific practice (First edition). Oxford, United Kingdom: Oxford University Press.

Illari, P., \& Russo, F. (2016). Information Channels and Biomarkers of Disease. Topoi, 35(1), 175-190. https://doi.org/10.1007/s11245-013-9228-1

International Programme on Chemical Safety (Ed.). (2001). Biomarkers in risk assessment: validity and validation. Geneva: World Health Organization.

Joffe, M., Gambhir, M., Chadeau-Hyam, M., \& Vineis, P. (2012). Causal diagrams in systems epidemiology. Emerging Themes in Epidemiology, 9(1), 1.

https://doi.org/10.1186/1742-7622-9-1

Kelly, M. P., Kelly, R. S., \& Russo, F. (2014). The Integration of Social, Behavioral, and Biological Mechanisms in Models of Pathogenesis. Perspectives in Biology and Medicine, 57(3), 308-328. https://doi.org/10.1353/pbm.2014.0026

Kelly, M. P., \& Russo, F. (2017). Causal narratives in public health: the difference between mechanisms of aetiology and mechanisms of prevention in non-communicable diseases. Sociology of Health \& IIIness, 40(1), 82-99. https://doi.org/10.1111/1467$\underline{9566.12621}$

Kelly-Irving, M., \& Delpierre, C. (2017). The Embodiment Dynamic over the Life Course: A Case for Examining Cancer Aetiology. In C. Meloni, J. Cromby, D. Fitzgerald, \& S. Lloyd (Eds.), The Palgrave Handbook of Biology and Society (pp. 519-540). London: Palgrave.

Kelly-Irving, M., Lepage, B., Dedieu, D., Lacey, R., Cable, N., Bartley, M., ... Delpierre, C. (2013). Childhood adversity as a risk for cancer: findings from the 1958 British birth cohort study. BMC Public Health, 13(1). https://doi.org/10.1186/1471-2458-13-767 Krieger, N. (2001). A glossary for social epidemiology. Journal of Epidemiology \& Community Health, 55(10), 693-700. https://doi.org/10.1136/jech.55.10.693

Krieger, N. (2008). Proximal, Distal, and the Politics of Causation: What's Level Got to Do With It? American Journal of Public Health, 98(2), 221-230. https://doi.org/10.2105/AJPH.2007.111278 
Kronfeldner, M. (2014). Commentary: How norms make causes. International Journal of Epidemiology, 43(6), 1707-1713. https://doi.org/10.1093/ije/dyu130

Marmot, M. (2005). Social determinants of health inequalities. The Lancet, 365(9464), 1099-1104. https://doi.org/10.1016/S0140-6736(05)71146-6

McLean, G., Gunn, J., Wyke, S., Guthrie, B., Watt, G. C., Blane, D. N., \& Mercer, S. W. (2014). The influence of socioeconomic deprivation on multimorbidity at different ages: a cross-sectional study. British Journal of General Practice, 64(624), e440-e447. https://doi.org/10.3399/bjgp14X680545

Miller, G. E., Chen, E., \& Parker, K. J. (2011). Psychological stress in childhood and susceptibility to the chronic diseases of aging: Moving toward a model of behavioral and biological mechanisms. Psychological Bulletin, 137(6), 959-997. https://doi.org/10.1037/a0024768

Muntaner, C. (2013). Invited Commentary: On the Future of Social Epidemiology--A Case for Scientific Realism. American Journal of Epidemiology, 178(6), 852-857. https://doi.org/10.1093/aje/kwt143

Purewal, S. K., Marques, S. S., Koita, K., \& Bucci, M. (2016). Assessing the Integration of the Center for Youth Wellness Adverse Childhood Experiences Questionnaire (CYW ACEQ) in a Pediatric Primary Care Setting. Journal of Adolescent Health, 58(2), S47. https://doi.org/10.1016/j.jadohealth.2015.10.106

Russo, F. (2009). Causality and causal modelling in the social sciences: measuring variations. Dordrecht: Springer.

Russo, F., \& Williamson, J. (2012). EnviroGenomarkers: The Interplay Between Mechanisms and Difference Making in Establishing Causal Claims. Medicine Studies, 3(4), 249262. https://doi.org/10.1007/s12376-012-0079-7

Salmon, W. C. (1984). Scientific explanation and the causal structure of the world. Princeton, N.J: Univ. Pr.

Sapsford, R. (2006). Indicator. In V. Jupp (Ed.), The Sage Dictionary of Social Research Methods. London: SAGE Publications Ltd.

Sauer, P. J. J., Nicholson, A., \& Neubauer, D. (2016). Age determination in asylum seekers: physicians should not be implicated. European Journal of Pediatrics, 175(3), 299303. https://doi.org/10.1007/s00431-015-2628-z 
Schulte, P. A. (1993). A conceptual and historical framework for molecular epidemiology. In P. A. Schulte \& F. P. Perera (Eds.), Molecular epidemiology: principles and practices. San Diego: Academic Press.

Schulte, P. A., \& Perera, F. P. (Eds.). (1993). Molecular epidemiology: principles and practices. San Diego: Academic Press.

Tietjen, G. E., Khubchandani, J., Herial, N. A., \& Shah, K. (2012). Adverse Childhood Experiences Are Associated With Migraine and Vascular Biomarkers. Headache: The Journal of Head and Face Pain, 52(6), 920-929. https://doi.org/10.1111/i.15264610.2012.02165.x

Velupillai, Y., Packard, C. J., Batty, G. D., Bezlyak, V., Burns, H., Cavanagh, J., ... Tannahill, C. (2008). Psychological, social and biological determinants of ill health (pSoBid): Study Protocol of a population-based study. BMC Public Health, 8(1). https://doi.org/10.1186/1471-2458-8-126

Vineis, P. (1997). Proof in observational medicine. Journal of Epidemiology and Community Health, 51(1), 9-13.

Vineis, P., Avendano-Pabon, M., Barros, H., Chadeau-Hyam, M., Costa, G., Dijmarescu, M., ... Stringhini, S. (2017). The biology of inequalities in health: the LIFEPATH project. Longitudinal and Life Course Studies, 8(4). https://doi.org/10.14301/Ilcs.v8i4.448

Vineis, P., \& Chadeau-Hyam, M. (2011). Integrating biomarkers into molecular epidemiological studies: Current Opinion in Oncology, 23(1), 100-105. https://doi.org/10.1097/CCO.0b013e3283412de0

Vineis, P., Chadeau-Hyam, M., Gmuender, H., Gulliver, J., Herceg, Z., Kleinjans, J., ... Wild, C. P. (2017). The exposome in practice: Design of the EXPOsOMICS project. International Journal of Hygiene and Environmental Health, 220(2), 142-151. https://doi.org/10.1016/j.ijheh.2016.08.001

Vineis, P., Chatziioannou, A., Cunliffe, V. T., Flanagan, J. M., Hanson, M., Kirsch-Volders, M., \& Kyrtopoulos, S. (2017). Epigenetic memory in response to environmental stressors. The FASEB Journal, 31(6), 2241-2251. https://doi.org/10.1096/fj.201601059RR

Vineis, P., Illari, P., \& Russo, F. (2017). Causality in cancer research: a journey through models in molecular epidemiology and their philosophical interpretation. Emerging Themes in Epidemiology, 14(1). https://doi.org/10.1186/s12982-017-0061-7 
Vineis, P., \& Kriebel, D. (2006). Causal models in epidemiology: past inheritance and genetic future. Environmental Health, 5(1). https://doi.org/10.1186/1476-069X-5-21

Vineis, P., \& Perera, F. (2007). Molecular Epidemiology and Biomarkers in Etiologic Cancer Research: The New in Light of the Old. Cancer Epidemiology Biomarkers \&amp; Prevention, 16(10), 1954-1965. https://doi.org/10.1158/1055-9965.EPI-07-0457

Walker, S. E., \& Carrion, V. G. (2015). The Center for Youth Wellness: A Community-Based Approach to Holistic Health Care in San Francisco. In L. Roberts, D. Reicherter, J. Adelsheim, \& S. V. Joshi (Eds.), Partnerships for mental health: narratives of community and academic collaboration (pp. 37-51). Cham: Springer.

Wilkinson, R., \& Marmot, M. (Eds.). (2003). The solid facts: social determinants of health (2nd ed). Copenhagen: WHO Regional Office for Europe. 